\title{
Bodenrechte in den Chittagong Hill Tracts
}

Die nord-südlich verlaufenden Bergketten im Osten von Chittagong (am Golf von Bengalen) wurden 1860 unter britische Kolonialverwaltung genommen, 1947 dem östlichen Flügel Pakistans zugeschlagen und gehören deshalb, seit dem Unabhängigkeitskrieg 1971, zu Bangladesh. Entgegen den bei der Teilung Britisch-Indiens zugrunde gelegten Prinzipien, stellte die muslimische Bevölkerung der Chittagong Hill Tracts 1947 nur eine Minderheit von höchstens 6\% dar (1951 18000 Muslim, gegenüber 215000 Buddhisten, 41000 Hindu, 4000 Christen und 9000 «tribals», vgl. Census 1961: IV-40). Die Kultur der Bewohner der Hill Tracts unterschied sich grundlegend von derjenigen der Bengalen der Ebene. Wenn irgendwo die Grenze zwischen Vorder- und Hinterindien zu ziehen war, dann hier entlang der Grenze zwischen dem Chittagong-Distrikt und den Hill Tracts, zwischen den indiden Bengalen indoeuropäischer Sprache mit Wasserreisbau in der Ebene und den palämongoliden Bergleuten mit Sprachen, die der tibetoburmanischen Familie anzuschließen sind (nur die Chakma sprechen einen bengalischen Dialekt, haben aber eine eigene, der burmanischen verwandte Schrift), deren Nahrungsgrundlage vorwiegend der Trockenreisanbau auf Schwendfeldern ist und deren nächste Verwandte in Tripura, in Arakan und im heutigen Mizoram leben. Geographisch mögen die Berge als das Hinterland der Chittagong-Ebene betrachtet werden - alle Flüsse der Berge münden, nachdem sie die Ebene durchquert haben, im Golf von Bengalen -, aber genau so gut kann man den Ebenen-Distrikt, der noch von den westlichsten, niedrigsten Hügelketten durchzogen wird, als Vorland der Berge betrachten, so $\mathrm{da} \beta$ die scharfe kulturelle Grenze nicht einfach als eine solche zwischen Ebenen- und Bergbewohnern erklärt werden kann. In der Tat war zumindest der Südteil der Ebene noch im 18. Jahrhundert kein Bengalen-Land, und erst im 17. Jahrhundert kam die Stadt Chittagong endgültig unter Muslim-Herrschaft, nachdem den Muslim-Herrschern zuvor neben den Tripura vor allem die Arakaner den Besitz streitig gemacht hatten (vgl. SYED MURTAZA ALI 1964).

Arakanischer Provenienz sind die Marma (sprachlich von der gleichen Ausgangsform, herzuleiten wie «Burma»), die nicht nur in den Fluftälern der nord- westlichen und vor allem der südlichen Hill Tracts wohnten, sondern ebenso im südlichen Ebenen-Vorland. Bewohner des südlichen Chittagong-Distrikts wie auch des nördlichen Arakan waren auch die Sak, die vor 30 Jahren im Süden der Hill Tracts nur noch etwa tausend Leute zählten, deren Vorfahren jedoch, zusammen mit Marma und Tippera, die ihre Sprache wechselten, zur Entstehung jener Ethnie beigetragen haben mögen, die heute als Chakma die Hauptbevölkerung der mittleren und nördlichen Hill Tracts stellt (vgl. LÖFFLER 1964, BERNOT 1967). In dem an das Chittagong-Gebiet nördlich anschlieBenden Tripura fanden sich die (eine tibetoburmanische Sprache sprechenden) Tippera bereits in der Mitte des 19. Jahrhunderts nur noch im östlichen Hügelland; in der westlich vorgelagerten, von Bengalen bewohnten Ebene spielte der Raja von Tripura nur noch die Rolle eines Großgrundbesitzers (vgl. HUNTER 1876). Inzwischen bilden die Bengalen aber auch im ehemaligen Tripura Hill State die Majorität.

Da die Briten dem Raja von Tripura als Hindu-Herrscher seinen eigenen Staat zuerkannt hatten, wurde Tripura bei der Teilung 1947 Indien zugeordnet; den Rajas der Chakma und Marma in den Chittagong Hill Tracts hingegen wurde eine entsprechende Anerkennung nicht zuteil, obwohl ihnen unter den Moghul-Herrschern ein ähnlicher Status zugekommen sein mag wie dem Tripura-Raja, indem sie sich zu Tributleistungen verpflichteten, die im Falle der Chittagong Hill Tracts vorwiegend aus Baumwolle bestanden. Als Gegenleistung wurde den Bergleuten der Zugang zu den Märkten der Ebene gewährt (LEWIN 1870: 34).

Bemerkenswerterweise residierten weder der Chakma-Raja noch der Bohmong genannte MarmaRaja im Berggebiet, sondern dort, wo die beiden Hauptflüsse, der Kornofuli und der Songu, in die Ebene eintraten; erst zur britischen Kolonialzeit verlegten die Rajas ihre Residenz weiter flußaufwärts in das Gebiet der Hill Tracts (HUTCHINSON 1909: 23, 29). Und erst die Briten ordneten ihnen

Lorenz G. Löffler, Prof. Dr., Ethnologisches Seminar, Freiensteinstrasse 5, 8032 Zürich 
Ende des letzten Jahrhunderts ein Territorium zu, genannt "Circle», bei dessen Einwohnern ihnen die Steuereinziehung oblag. Wie weit ihre Macht zuvor reichte, ist unklar. Manches spricht dafür, daß es auBer den von den Briten zu territorialen Chiefs erklärten Rajas noch weitere gab (BERNOT 1967: 87ff.). Der Mong-Raja der nördlichen Marma erhielt diese Rolle erst nachträglich durch Ausgliederung eines eigenen Circles aus dem Chakma-Circle. Bekannt ist, daß die Gruppe der Berg-Chakma, die sogenannten Tongcengya oder Dengnak, sich konstant weigerten, den Chakma-Raja als ihr Oberhaupt anzuerkennen und ihm dementsprechend $\mathrm{Ab}$ gaben zu liefern, und die Tippera und Marma der nördlichen Hill Tracts, die dem Chakma-Chief unterstellt wurden, dürften dies ebensowenig getan haben.

In den südlichen Hill Tracts finden sich neben den Marma in den Haupttälern weitere Ethnien in den Nebentälern und auf den Bergen: Mru, Khumi, Khyang und Bawm (und in den mittleren Hill Tracts, den letzteren sprachlich nahestehend, die Pangkhua), von denen nur die Bawm sozial stratifiziert waren und ihren eigenen Raja hatten (vgl. SPIELMANN 1968), während Mru, Khumi und Khyang nur einzelne Sippen- oder Dorfchefs kannten. Bei ihnen zeichnete man sich als großer Mann aus, indem man Verdienstfeste gab, und nicht, indem man Tribut kassierte. Entscheidend für die Entwicklung der Bodenrechte waren jedoch nicht die Verhältnisse bei den kleineren Berg-Ethnien, sondern die bei den großen Ethnien der Täler, d. h. vor allem Chakma und Marma.

Ich spreche absichtlich von Bodenrechten im Plural, denn es gibt zwei Konstrukte, die unterschieden werden müssen: das der Feldbauern einerseits und das der staatlichen Machthaber andererseits. Beginnen wir mit dem Rechtssystem der Feldbauern, wobei es meines Wissens prinzipiell keinen Unterschied macht, um welche Ethnie es sich handelt. In diesem System hat jeder Mensch, ob männlich oder weiblich, ledig oder verheiratet, das Recht auf Bodennutzung, sei es zum Errichten einer Behausung, zur Anlage eines Feldes oder einfach zur Nutzung von Erde und Wasser, Fauna und Flora, d. h. zum Sammeln und Jagen. Dieses Recht kann der einzelne jedoch nicht an jedem beliebigen, ihn gutdünkenden Ort ausüben; vielmehr braucht er dazu die Zustimmung seiner Umwelt, d. h. seiner Mitmenschen einerseits und der Natur andererseits. Letztere hat durchaus die Möglichkeit, ihm Zustimmung oder Ablehnung zu bekunden; wer der Ablehnung zuwider handelt, muß mit Krankheiten oder Mißernten rechnen. Es gibt gewisse Stellen im Boden, z. B. Sickerplätze, deren Nutzung dem Menschen untersagt ist, so daß sie sich $\mathrm{z}$. B. auch nicht inmitten eines Feldes befinden dürfen. Auf nicht solcherart ausgeschlossenen Territorien hingegen hat man zunächst ein Zeichen aufzustellen und den Traum der folgenden Nacht zu beobachten: ist seine Symbolik positiv, steht einer Nutzung zunächst nichts im Wege; für eine ungefährdete weitere Feldarbeit und einen guten Ertrag sind im weiteren Verlauf des Schlagens, Brennens, Säens, Jätens und Erntens jedoch bestimmte Opferzeremonien nötig, um das Wohlwollen der zuständigen Naturkräfte zu gewinnen. Was hier für die Anlage eines Feldes gesagt wurde, gilt ebensogut für die Anlage eines Dorfes. Sind bereits die Traumzeichen negativ, sollte man sich einen neuen Platz suchen; durch zunehmende Bodenknappheit gezwungen, muß man sich unter Umständen mit einer leichten Verschiebung begnügen, wie überhaupt manche alte Regel zunehmend mißachtet wird, obschon die abnehmenden Felderträge deren Richtigkeit im nachhinein bestätigen. In der Tat bestellt niemand aus freien Stücken einen nur geringen Ertrag versprechenden Boden. Und es ist niemandem zu verdenken, daß er die beste Feldfläche möglichst nahe beim Dorf nutzen möchte. Dafür, daß sich hier nicht alle in die Quere kommen, sorgt die Dorfversammlung der Haushaltsvorstände, d. h. im wesentlichen der verheirateten Männer. Daß dabei die Interessen der Ledigen und Frauen etwas schlechter wegkommen, kann nicht ausgeschlossen werden.

Da der Feldbau, wie er auf den Hängen der Hill Tracts betrieben wird, mehrjährige Brachzeiten erfordert, hat die Dorfversammlung im Prinzip zwei Optionen: entweder wird jährlich rotierend ein ganzes Segment der Dorfflur zur Bestellung bestimmt, das dann in einzelne Felder parzelliert wird. Der Vorteil ist, daß auf diese Art gegenseitige Hilfe sehr leicht ist, und es somit auch jeder gleich weit hat; der Nachteil jedoch ist, daß eine so große zusammenhängende Schwendfläche das ökologische Gleichgewicht stärker stört und gegen Ernteschäden viel anfälliger ist als kleine, über die ganze Flur verstreute Einzelfelder. Diese Art der Anlage ist die andere Option, die in den Hill Tracts die übliche ist. Die Diskussion über die Verteilung wird hier dadurch vereinfacht, daß im Prinzip der frühere Nutzer bevorrechtigt ist, wenn es darum geht, eine brachliegende Feldfläche wieder zu nutzen. Nur ändern sich über die Jahre hinweg die Anzahl und Größe der einzelnen Familien; wer weniger braucht, könnte die Brachzeit ausdehnen, aber da sind immer andere, die mehr brauchen, und so gilt das Vorrecht nur dann, wenn zwei Haushalte den gleichen Platz für ihr Feld beanspruchen. Wer eine Fläche bestellen will, ehe der frühere Nutzer darauf zurückkommen möchte, kann nicht daran gehindert werden (vgl. auch BERNOT 1967: 225-45; MEY 1979: 101-106).

Auf geschlossenen Widerstand trifft nur der, der angesichts zunehmender Landknappheit ein neues Feld dort anlegen möchte, wo sich bisher die Holz- 


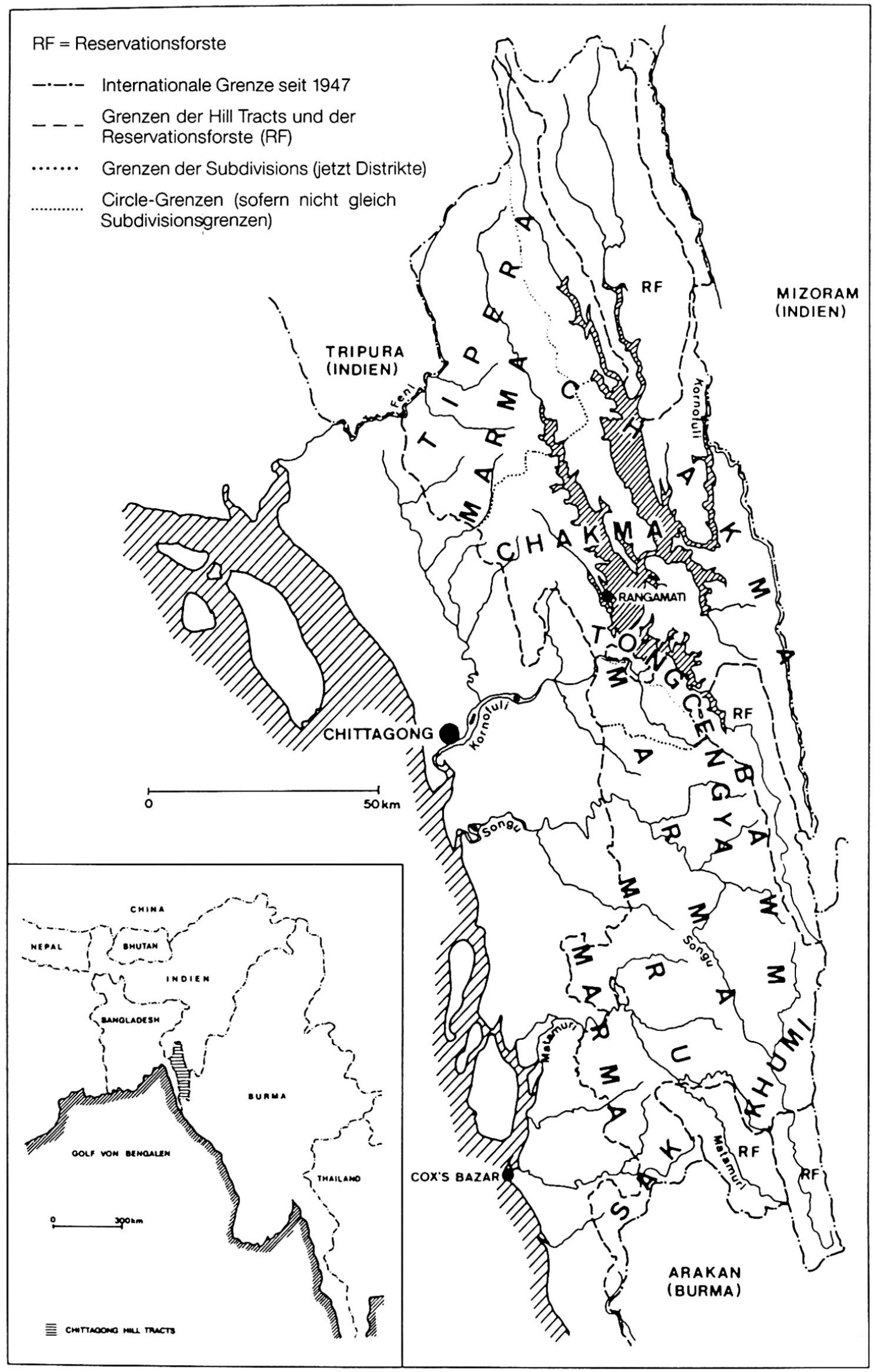

Abb. 1 Karte zur Lokalisation der Ethnien um 1960 (aus «Bedrohte Zukunft», Völkerkundemuseum Zürich, 1988) 
und Bambusreserven des Dorfes befanden. Wer den Mut hat, sich darüber hinwegzusetzen, kann aber auch daran nicht gehindert werden, obwohl es allen im Dorf letztlich teuer zu stehen kommt, da man nun Bauholz und Bambus von weit her holen und dazu in den Nachbardörfern um Genehmigung einkommen muß, die versagt werden kann. Und geschädigt fühlten sich die Leute im Nachbardorf auch, wenn man auf ihrer Flur ein Feld anlegt, ohne dazu die Zustimmung eingeholt zu haben - solche Übergriffe können zu Gerichtsverhandlungen beim Headman führen (vgl. BERNOT 1967: 228-29). Dies zeigt, daß - obschon wohl erst mit zunehmender Bodenverknappung - sich ein gewisser Kommunalbesitz am Dorfterritorium herausgebildet hat, dessen Grenzen, obwohl auf keiner Karte verzeichnet, den Dörflern wohl bekannt sind. Diese Dorfgrenzen sind nicht identisch mit den von der kolonialen Verwaltung gezogenen Grenzen der sogenannten Mouzas; diese umfassen in der Regel mehrere Dörfer, können diese aber auch, z. B. wenn sie auf einem Berggrat oder zu beiden Seiten eines Baches liegen, entzweischneiden. Dörfer bestehen fast immer nur aus Angehörigen einer Ethnie; die Mouza-Grenzen hingegen nehmen auf ethnische Zugehörigkeit keine Rücksicht.

Dennoch war für die ersten Mouzas das Dorf das Vorbild, aber nicht in Form der kleinen Weiler in den Bergen, sondern der großen Siedlungen in den Tälern. In diesen amteten von den Rajas eingesetzte Magistraten, zu deren Aufgaben neben der Rechtswahrung vor allem die Steuereintreibung für den Raja gehörte, von der sie zum Teil auch selbst profitierten. Bei den Marma hießen sie Ruatsa («Dorf-Esser»); ihnen unterstanden auch die Weiler, die sich als Ableger der Dörfer gebildet hatten; doch zu zahlen hatten die Dörfler ihre Abgaben nicht in jedem Fall an den Ruatsa ihres Dorfes, sondern an den, aus dem ihre Frau stammte: die Abgaben waren mithin keine Steuern für die Nutzung des lokalen Bodens (BERNOT 1967: 109). Bei den Chakma wurden die entsprechenden Agenten «Dewan» genannt. Diese waren einst Sippenchefs (LEWIN 1870: 91); noch vor 1860 wurde es jedoch üblich, diese Posten zu auktionieren, d. h. als blosse Steuereinnehmerposten an den Meistbietenden zu verkaufen. "The rights of the head-man that were thus frequently put up for sale under the authority of British officers were rights affecting human beings, and although termed táluks, they had no connection with any form of land tenure» schreibt HUNTER 1876 (Bd. 6, S. 91).

In den gegen Ende des 19. Jahrhunderts von den Engländern geschaffenen Mouzas avancierte ein Teil der alten Ruatsa und Dewan zu Headmen, doch blieben ihre Funktionen im wesentlichen die gleichen, d.h. neben der lokalen Rechtsprechung und Streitschlichtung oblag ihnen auch der Einzug und die Weiterleitung der Abgaben an den Raja (jetzt:
Chief), nur waren die Abgaben jetzt in Geld zu entrichten, und zwar an den Headman, in dessen Mouza man wohnte. Neu war aber insbesondere, da $\beta$ diese Abgaben jetzt als «jhum tax» bezeichnet wurden, d. h. als «Feldsteuer» («jhum» ist die bengalische Bezeichnung für ein Schwendfeld). Diese Steuer bemaß sich allerdings nicht nach der Größe des genutzten Areals und wurde, wie schon die entsprechende vorkoloniale "capitation tax», auch nur solchen Haushalten auferlegt, die eine vollständige Familie bildeten und ihren Lebensunterhalt nicht aus anderen Beschäftigungen zogen (HUNTER 1876: 88).

Die Interpretation als Feldsteuer geht auf eine Abmachung der Briten mit den Chakma am Ende des 18. Jahrhunderts zurück. «In 1791 the Board authorized the Collector of Chittagong to fix a moderate cash payment, and the cotton tax was abolished. This settlement ist known as Jum Bangu or right to Jum under a chief and was in the name of Jan Baksh: the annual rent to be paid being fixed as Rs. 1,775" (HUTCHINSON 1909: 24). Wohl konnte es den Briten damals noch ziemlich gleichgültig sein, wie der Raja durch Abgaben seiner Untertanen zu seinem Geld kam, nach der Übernahme der Verwaltung jedoch war die «jhum tax» zu regulieren. Noch nach 1900 erklärt Hutchinson, der damalige oberste Verwaltungsbeamte (Superintendent der Hill Tracts): "This tax is tribute payable to the state, it in no way partakes of the nature of rent, or bears any relation to the land cultivated» (HUTCHINSON 1909: 93). Doch schon vor 1875 bestätigten Gerichtsurteile, daß diese Steuer nicht mehr durch ethnische, sondern durch territoriale Zugehörigkeit bestimmt war: Chakma, die die Hill Tracts verlassen hatten, waren ihrem Chief nicht mehr steuerpflichtig (HUNTER 1876: 89). Aus der Kopfsteuer wurde, gewissermassen unbeabsichtigt, eine Steuer für die Nutzung des Bodens, den sich die britische Krone angeeignet hatte und für dessen Nutzung durch die Einheimischen jetzt die Headmen und Chiefs als Steuereinzieher zu fungieren hatten. 50 Jahre nach HUTCHINSON schreibt BESSAIGNET (1958: 38): "As was confirmed to me by the Board of Revenue, Dacca, revenue is assessed on the jhum field, not on the household."

Andererseits stellt BESSAIGNET (1958: 37) fest: «The ordinary tribesman does not own the land but, according to tribal notions, he has an inviolable right to use whatever land he sees fit to occupy." Und der oben zitierte Satz Hutchinsons läßt keinen Zweifel daran, daß die Landnutzungsrechte von der «jhum tax» offiziell unberührt blieben. Doch gaben sich die Briten zunächst alle Mühe, den Bewohnern der Hill Tracts ihren «Nomadismus» abzugewöhnen. In einem Fünftel des gesamten Territoriums der Hill Tracts wurde der Schwendbau überhaupt verboten; die entsprechenden Areale wurden 1880-83 zu Reservationsforsten erklärt. In den anderen Gebieten mußten sich die Einwohner unter einem Headman 


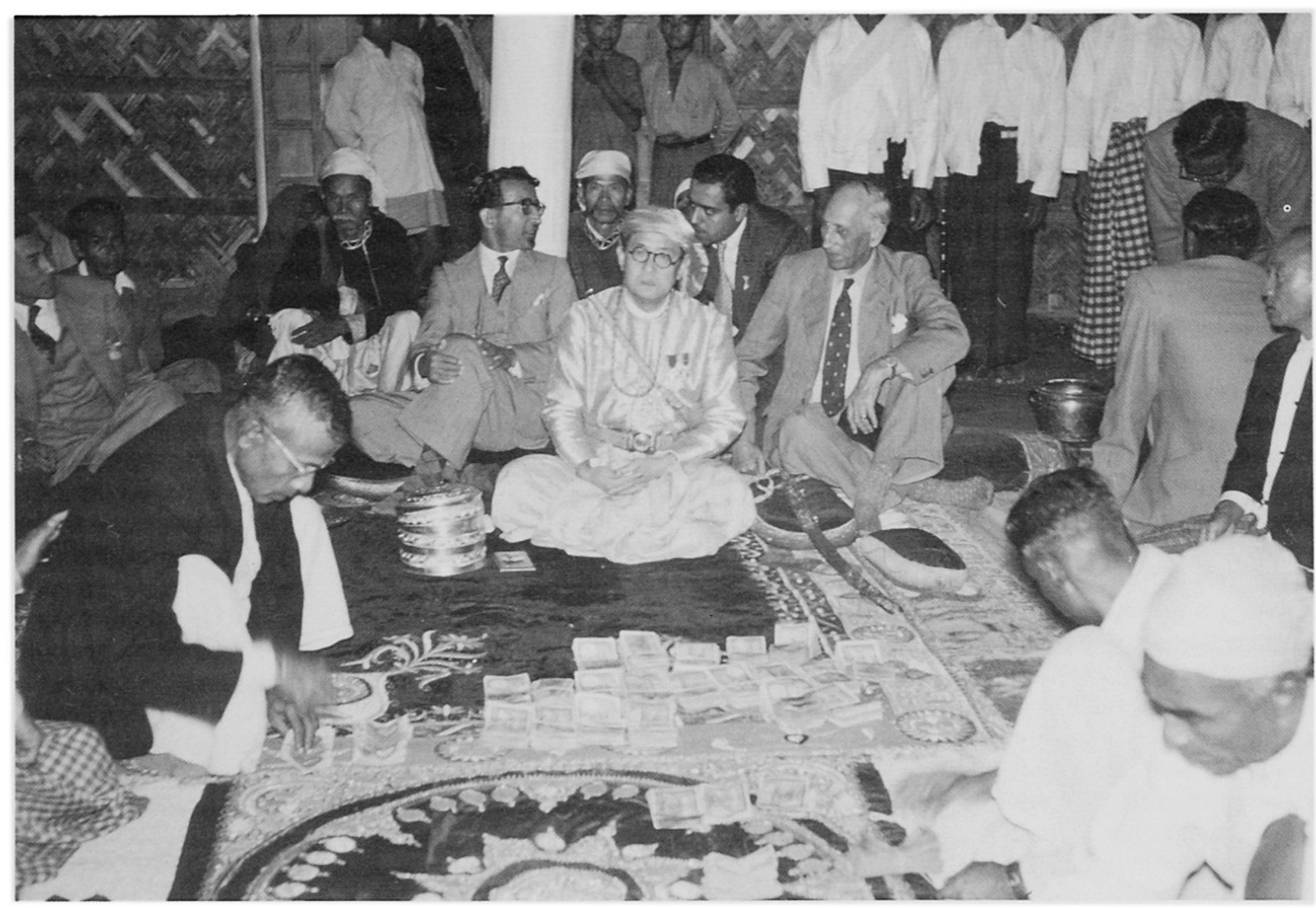

Abb. 2 Das koloniale System im Übergang (1955): Die Headmen liefern dem Chief (hier dem Bohmong-Raja) die Feldsteuern ab, der sie, nach Abzug seines Anteils, über den Deputy Commissioner (als obersten Verwaltungsbeamten der Hill Tracts, damals noch ein Engländer, zur Linken des Chiefs) an die Regierung weiterleitet (hier vertreten durch den Commissioner des Chittagong Districts, einen Bengalen, zur Rechten des Chiefs).

registrieren lassen, und jeder, der außerhalb seiner Mouza ein Feld anlegte, hatte fortan nochmal die halbe jhum tax zu bezahlen - eine Bestimmung, die den Landnutzungsbezug dieser Steuer nochmals verdeutlicht (vgl. MEY 1980: 130).

Noch lieber hätten die Briten es allerdings gesehen, wenn die Bewohner der Hill Tracts sich vom Schwendbau überhaupt abgewandt hätten, zum Pflugbau übergegangen wären und ihren Landbesitz hätten registrieren lassen. HUNTER (1876: 91-92) schreibt dazu: «. . . it appears that up to the present date, the attempt to make the hill-men give up their nomadic form of cultivation has met with a very limited amount of success. The chiefs and head-men energetically opposed the change at the first attempt to introduce it, and some of them even applied for leases of large tracts of land with no other object than to prevent any other hill-men obtaining the lands. "Was Hunter nicht sah, war die Hürde der hohen Investitionen, die zu überwinden war, wollte man ein Feld dauerhaft kultivierbar machen; am Ende des Jahrhunderts half die Regierung mit Anleihen zum Erwerb von Ackerbaugeräten und Zugtieren nach, und die Bevölkerungszunahme in den Tälern tat ein übriges, um den Übergang zum Pflugbau, wo immer ein Stück Flachland zur Verfügung stand, zu beschleunigen. Interessant bleibt jedoch an Hunters Bemerkung, daß die Headmen sich veranlaßt sahen, Boden zu «leasen», d. h. sich mit Zahlungen an die Briten den bisher kommunalen Boden anzueignen und damit zugleich das neue Konstrukt anzuerkennen, nach dem sich alles Land im Besitz der britischen Krone befand. Bereits zuvor hatte die Chakma Rani versucht, sich als Grundbesitzerin ihres gesamten Territoriums registrieren und veranlagen zu lassen, dies wurde ihr jedoch nicht gestattet (HUTCHINSON 1909: 24-25).

Der größte Teil der Hill Tracts war und ist für den Pflugbau ungeeignet. Hier blieb denn auch die Schwendbautradition ungebrochen, obschon kein Zweifel daran bestehen kann, daß viele Marma und Chakma nichts dagegen gehabt hätten, zu Besitzern eines Wasserreisfeldes zu avancieren. Nach 1900 scheint auch das Bild, das sich die britische Verwaltung vom Schwendbau machte, etwas positiver geworden zu sein. HUTCHINSON (1909: 67) trug einige 
der ökonomischen Vorteile zusammen, die der Schwendbau auch der Verwaltung brachte; unbeachtet blieb jedoch lange der Unterschied in den sozialen Beziehungen, die die beiden Kultivationsformen mit sich bringen: die Differenzierung in Landeigentümer und landlose Arbeiter im Ackerbau und die soziale Gleichheit dort, wo, wie im Schwendbau der Hill Tracts, jedem der Zugang zum Land gewährleistet ist, wo Ausbeutung nur über Verschuldung möglich ist.

Die resultierenden krassen Unterschiede in Mentalität und Moral waren allerdings zu offenkundig (Lug und Betrug, Gaunerei und Diebstahl waren in den Hill Tracts, im Gegensatz zum Bengalen-Land, extrem selten), als da $\beta$ den Briten daran gelegen sein konnte, die Verhältnisse der Ebene auch in den Bergen zu verbreiten (vgl. LEWIN 1870). Nach ersten schlechten Erfahrungen Ende des 19. Jahrhunderts wurde deshalb den Hill Tracts im Jahr 1900 offiziell ein Sonderstatus zugestanden (vgl. Chittagong Hill Tracts Manual 1942). Fortan waren die einheimischen Bräuche und Normen wieder zu respektieren, und nicht hier Geborenen wurde es äußerst schwer gemacht, sich dauerhaft niederzulassen oder gar Land zu erwerben. Wohl wäre es möglich gewesen, auch Schwendbauland zu privatisieren, aber damit wäre es auch unvermeidlich geworden, daß solches Land eines Tages verpfändet und letztlich in die Hände eines bengalischen Geldleihers übergegangen wäre.

Dennoch ergab sich auch für die Schwendbauern mit der Verbreitung des Mandarinen-Anbaus ab 1940 zunehmend die Möglichkeit, Kommunalland de facto, wenn auch nicht de jure, zu privatisieren, indem sie auf solchem Land Plantagen anlegten. Gepflanzte Bäume galten schon immer, wie jedes Gepflanzte, als Privateigentum des Pflanzers und konnten auch vererbt werden, unabhängig vom Wohnsitz ihres Eigentümers. Erst der pakistanischen Regierung, die sich, gegen den Widerstand der zu Recht um ihre Privilegien bangenden Chiefs, bemühte, den Sonderstatus der Hill Tracts abzubauen, gefiel es, solche Obstplantagen als Privatland registrieren zu lassen. Und die Regierung Bangladeshs schließlich forderte die Schwendbauern auf, all ihr Land registrieren zu lassen, sei es als Privat-, sei es als Kommunalland. Was die Briten mit dem Pflugland begannen, sollte so 100 Jahre später mit dem Schwendland vollendet werden: gegen eine Gebühr an die Regierung allgemeine Nutzungsrechte am Boden in Eigentumsrechte umzuwandeln. Nicht unbedingt zum Schaden derjenigen, die sich dazu bereitfanden: denn im Proze $\beta$ der nun folgenden Einsiedlung von Bengalen mußten die bisherigen Eigentümer - zumindest de jure, wenn auch nicht immer de facto - entschädigt werden und hatten Anrecht auf Rehabilitation; diejenigen jedoch, die ihr «Eigentum» nicht hatten registrieren lassen, konnten einfach vertrieben werden, sie hatten, nach dem staatlichen Konstrukt, keine Bodenrechte, sie okkupierten nur, illegal, Regierungsland.

Um dem Konstrukt der Illegalität auch eine gesetzliche Grundlage zu verschaffen, tat die Regierung ein übriges: sie verbot, 1986, kurzerhand den Schwendbau in den Hill Tracts; 1870 hatten die Briten das gleiche Verbot erlassen, damals allerdings für den «regulation district», d. h. die ChittagongEbene mit ihren niedrigen Hügelketten. Damals betraf es eine (unbekannte) Anzahl Schwendbauern, die sich nach Arakan oder in die Hill Tracts (die damals weniger als 100000 Einwohner zählten) zurückzogen; heute aber betrifft es mindestens 300000 Schwendbauern, denen die Existenzgrundlage verboten wird und die als solche nur weiterexistieren können, wenn sie nach Burma (Arakan oder Chin Hills) emigrieren; denn in Indien werden Flüchtlinge aus den Hill Tracts nur in Lagern gefangengehalten. Die Alternative ist, sich von der Regierung Bangladeshs, gegen eine für manche unerschwingliche Gebühr, zwei Hektar Boden zuschreiben zu lassen, in Dörfern, in denen ethnische Zugehörigkeiten keine Rolle mehr spielen dürfen, und sich zusammen mit landlosen bengalischen Umsiedlern aus der Ebene von Regierungsbeamten in der Kunst des Anbaus von Cashcrops unterweisen zu lassen, um dafür eine Zeitlang mit Subventionen am Leben erhalten zu werden.

$\mathrm{Da} B$ den Feldbauern auf diese Art in wenigen Jahren ein besseres Einkommen als je bisher beschert sein wird, steht im Programm einer kanadischen Expertenkommission, die den Plan für den CashcropAnbau 1964-66 ausarbeitete (FORESTAL 1966), aber auch, da $\beta$ diese Prognose von Randbedingungen abhängig ist, die bis heute nicht gegeben sind. Auch hielt die Kommission bestenfalls $20 \%$ des Landes für solcherart nutzbar: die restliche Bevölkerung sei in der Forstwirtschaft zu beschäftigen. Die bengalischen Experten entdeckten inzwischen, daß auch das ganze restliche Bergland nutzbar gemacht werden könnte, nämlich für den Anbau von Gummibäumen. Da dafür aber den Einheimischen wie den Eingesiedelten jegliche Kenntnis fehlt, setzt die Regierung auf die Privatinitiative von Großunternehmern der bengalischen Mittelschicht: sie erhalten, wenn sie nur wollen, Tausende von Hektaren. Davon, da $\beta$ sie inzwischen rentable Gummiplantagen etabliert hätten, ist nichts bekannt, ebensowenig finden sich Angaben darüber, was mit der dort bisher ansässigen Bevölkerung geschehen soll bzw. geschieht (vgl. MUHAMMAD MUFAZZALUL HUQ 1982). $\mathrm{Da} B$ ein solches Überrollen des indigenen Bodenrechts durch das staatliche nicht ohne Widerstand seitens der betroffenen Bevölkerung bleiben würde, war vorauszusehen. Durch einen noch in der pakistanischen Zeit errichteten Staudamm des Kor- 
nofuli ging $1 / 5$ der einheimischen Bevölkerung der Hill Tracts ihres Bodens verlustig (SOPHER 1963); betroffen waren vor allem die Chakma. Ein paar tausend emigrierten auf der Suche nach neuem Land bis an den Fuß des Himalaya; die anderen versuchten zu überleben, indem sie sich die alte Regel zunutze machten, nach der jedem der Zugang zum Boden offen steht, d.h., indem sie in bereits bestehende Dörfer zogen, damit aber den Druck auf das verbliebene Land so erhöhten, da $B$ die eigentlich nötigen Brachezeiten radikal verkürzt werden mußten und in der Folge die Bodenfruchtbarkeit völlig zerstört wurde. Aber erst als die Regierung (vor allem in den verbliebenen fruchtbaren Gebieten, in denen auch Pflugbau möglich war) die Einsiedlung von landlosen Bengalen forcierte, formte sich in größerem Maße bewaffneter Widerstand. Die Regierung antwortete mit einer Verstärkung des Militärs und der paramilitärischen Kräfte bis hin zu einer Bewaffnung der Einsiedler, welche ihrerseits ihre einheimischen Nachbarn überfielen und massakrierten. Jeder erfolgreichen Operation des Widerstandes folgt eine grausame Repression, die die einheimische Zivilbevölkerung trifft und einen neuen Flüchtlingsstrom in Bewegung setzt (vgl. u. a. MEY 1984, BURGER \& WHITTACKER 1984, ORGANISING COMMITTEE 1986, AMNESTY INTERNATIONAL 1986, BRAUNS \& LÖFFLER 1986, MEY 1988, LÖFFLER 1988). Widerstand und Flucht aber zeigen die Unbrauchbarkeit des staatlichen Konstrukts: das koloniale Erbe der territorialen Oberhoheit legte der Regierung Bangladeshs nicht auch zugleich das Vorrecht in den Schoß, der einheimischen Bevölkerung das traditionelle, als unverletzlich betrachtete Recht auf freien Zugang zum Boden abzusprechen. Die britischen Chittagong Hill Tracts Regulations von 1900 gestanden das Recht der einheimischen Bevölkerung nicht $\mathrm{zu}$, sondern bestätigten es ihr nur. Leute, die keine Eigentümer des Landes sind, kann man nicht per Dekret enteignen; man kann nur das tun, was ihre Vorfahren schon in vorkolonialer Zeit gelegentlich taten: sie töten oder mit Gewalt vertreiben. Das, was die bengalische Regierung heute unternimmt, ist ein lokaler Eroberungskrieg im eigentlichen Sinn des Wortes: «Wir wollen das Land, nicht die Leute», soll ein hoher bengalischer Offizier gesagt haben. Sowenig sich auch die neuerdings deklarierte «Nation» der Bewohner der Hill Tracts als Bangladeshi fühlen mag, so schützt doch die kolonial ererbte territoriale Oberhoheit die Regierung Bangladeshs vor dem Vorwurf, einen internationalen Eroberungskrieg zu führen - aber sie schützt sie nicht vor dem Vorwurf des Völkermordes.

\section{Literatur:}

AMNESTY INTERNATIONAL (1986): Unlawful Killings and Torture in the Chittagong Hill Tracts. London

BERNOT, L. (1967): Les Paysans Arakanais du Pakistan Oriental. Paris: Mouton

BERNOT, L. (1967): Les Cak. Paris

BESSAIGNET, P. (1958): Tribesmen of the Chittagong Hill Tracts. Dacca

BRAUNS, C. D., und LÖFFLER, L. G. (1986): Mru. Bergbewohner im Grenzgebiet von Bangladesh. Basel: Birkhäuser

BURGER, J. and WHITTACKER; A. (Hrsg.) (1984): The Chittagong Hill Tracts. Militarization, Oppression and the Hill Tribes. London: Anti-Slavery Society

FORESTAL (1966): Chittagong Hill Tracts Soil and Land Use Survey 1964-1966 9 Vols. Vancouver

GOVERNMENT OF BENGAL BOARD OF REVENUE (1942): Chittagong Hill Tracts Manual. Alipore: Bengal Govt. Press.

HUNTER, W. W. (1876): A Statistical Account of Bengal. Vol. VI: Chittagong Hill Tracts, Chittagong, Noákháli, Tipperah, Hill Tipperah. London: Trübner

HUTCHINSON, R. H. SNEYD (1909): Chittagong Hill Tracts. Eastern Bengal and Assam District Gazetteers. Allahabad: Pioneer Press

LEWIN, T. H. (1870): Wild Races of South-Eastern India. London: W. H. Allen

LÖFFLER, L. G. (1964): Chakma und Sak. In: Internationales Archiv für Ethnographie 50, 1: 72-115

LÖFFLER, L. G. (Hrsg.) (1988): Bedrohte Zukunft. Bergvölker in Bangladesh. Zürich: Völkerkundemuseum

MEY, A. (1979): Untersuchungen zur Wirtschaft in den Chittagong Hill Tracts (Bangladesh). Bremen: Überseemuseum

MEY, W. (1980): Politische Systeme in den Chittagong Hill Tracts, Bangladesh. Bremen. Überseemuseum

MEY, W. (Hrsg.) (1984): “They are burning now village after village». Genocide in the Chittagong Hill Tracts, Bangladesh. Kopenhagen: IWGIA

MEY, W. (Hrsg.) (1988): “Wir wollen nicht euch - wir wollen euer Land." Macht und Menschenrechte in den Chittagong Hill Tracts, Bangladesh. Göttingen: Gesellschaft für bedrohte Völker

MUHAMMAD MUFAZZALUL HUQ (1982): Government Institutions and Underdevelopment. A Study of the Tribal Peoples of Chittagong Hill Tracts, Bangladesh. Birmingham: Institute of Local Government Studies

ORGANIZING COMMITTEE CHITTAGONG HILL TRACTS CAMPAIGN (1986): The Charge of Genocide. Human Rights in the Chittagong Hill Tracts. Amsterdam

SOPHER, D. E. (1963): Population Dislocation in the Chittagong Hills. In: Geographical Review 53: 337-362

SPIELMANN, H. J. (1968): Die Bawm-Zo. Heidelberg: Südasien-Institut

SYED MURTAZA ALI (1964): History of Chittagong. Dacca: Standard Publishers 